\title{
Editorial: Special Issue on Coding and Cryptography
}

\author{
Pascale Charpin* Thomas Johansson ${ }^{\dagger}$ \\ Gohar Kyureghyan ${ }^{\ddagger}$ Nicolas Sendrier* \\ Jean-Pierre Tillich*
}

Coding and cryptography remain of fundamental importance to the theory and implementation of telecommunication systems, computational systems, and secure networks. This special issue of Designs, Codes and Cryptography contains 28 journal refereed papers addressing almost all aspects of coding theory, cryptography and related areas, theoretical or applied. The selected papers are the full journal versions of extended abstracts accepted for presentation at the International Workshop on Coding and Cryptography, WCC 2015, held in Paris, France, April 2015. The variety of topics covered by WCC is a recurring feature of the workshop and attracts many colleagues, who work on several aspects of coding and cryptography.The topics covered by these 28 papers include coding theory, the design and analysis of cryptographic primitives, Boolean functions, the design of sequences and other related specializations within discrete mathematics that have applications to coding theory and/or cryptography.

Each of the 93 extended abstracts originally submitted to the workshop were reviewed by at least two members of the Program Committee. As a result of this screening process, 53 were selected for presentation at the workshop. The authors of the presented papers were in turn invited to submit full versions of their papers to the Designs, Codes and Cryptography journal. Each of the full-version submissions were once again thoroughly examined

\footnotetext{
*INRIA-Paris, Paris, France, Pascale.Charpin@inria.fr, Nicolas.Sendrier, Jean-Pierre.Tillich@inria.fr.

${ }^{\dagger}$ Lund University, Lund, Sweden, thomas.johansson@eit.lth.se.

†Otto-von-Guericke Universität Magdeburg, Germany, gohar .kyureghyan@ovgu . de.
} 
and commented upon by at least two anonymous reviewers. This volume is the end result of this second full-fledged reviewing process.

It remains to express our sincere thanks to the editorial staff of DCC for their continuous support. Thanks are also due to the authors for their contributions and to the large number of reviewers whose careful reading of these papers have ensured that this special issue is of the highest standard. 